\title{
Single prolonged stress induces dysfunction of endoplasmic reticulum in a rat model of post-traumatic stress disorder
}

\author{
BING XIAO ${ }^{1,2}$, BO YU $^{3}$, DONG-JUAN LIU ${ }^{1,2}$, FANG HAN $^{1,2}$ and YU-XIU SHI ${ }^{1,2}$ \\ ${ }^{1}$ Laboratory of Electron Microscopy, Department of Histology and Embryology, Basic Medical Sciences College; \\ ${ }^{2}$ Institute of Pathology and Pathophysiology, China Medical University, Shenyang, Liaoning 110001; \\ ${ }^{3}$ Department of Neurosurgery, Shengjing Hospital, China Medical University, Shenyang, Liaoning 110004, P.R. China
}

Received July 3, 2014; Accepted March 16, 2015

DOI: $10.3892 / \mathrm{mmr} .2015 .3590$

\begin{abstract}
The aim of the present study, was to investigate the involvement of the endoplasmic reticulum (ER) in post-traumatic stress disorder (PTSD) by detecting changes of ER chaperone protein 78 and ER-resident caspase 12 in the basolateral amygdala after exposure to single prolonged stress (SPS). The established rat model of PTSD was generated by exposure of the animals to SPS. The expression of glucose-regulated protein 78 (GRP78) was examined by immunofluorescence, western blot and reverse transcription-polymerase chain reaction (RT-PCR), and the expression of caspase 12 was examined by western blot and RT-PCR. The morphological changes of the ER were detected by transmission electron microscopy. The results showed that GRP78 expression significantly increased when compared to that in the control group 1 day after SPS exposure $(\mathrm{P}<0.05)$. The expression of caspase 12 was also significantly upregulated after SPS exposure and peaked at 7 days following SPS $(\mathrm{P}<0.05)$. Morphological evaluation showed that a tumescent ER, ER vacuolization and degranulation of the ER were present following SPS. In conclusion, the findings of the present study suggested that SPS induced GRP78 and caspase 12 upregulation and morphological changes of the ER in the amygdala, which may play important roles in the pathogenesis of PTSD rats.
\end{abstract}

\section{Introduction}

Post-traumatic stress disorder (PTSD) is an anxiety disorder that develops after exposure to a life-threatening traumatic experience. It is characterized by symptoms that often endure for years, including continuous re-experience of the traumatic

Correspondence to: Professor Yu-Xiu Shi, Laboratory of Electron Microscopy, Department of Histology and Embryology, Basic Medical Sciences College, China Medical University, 92 North 2nd Road, Shenyang, Liaoning 110001, P.R. China E-mail: shiyuxiu223@sina.com

Key words: single prolonged stress, post-traumatic stress disorder, endoplasmic reticulum, glucose-regulated protein 78 , caspase 12 event, avoidance of stimuli associated with the trauma, numbing of general responsiveness and increased arousal (1).

The endoplasmic reticulum (ER), a multifunctional signaling organelle, has a vital role in a variety of cellular functions, including post-translation modifications, synthesis and folding of membrane and secretory proteins, calcium sequestration for intracellular calcium homeostasis and apoptosis (2-4). Physiological and pathological stimuli that disrupt ER homeostasis can induce ER dysfunction or ER stress, including the accumulation of unfolded or misfolded proteins, oxidative stress, perturbation of calcium homeostasis and viral infection (5).

The 78-kDa glucose-regulated protein (GRP78), identical to the immunoglobulin heavy chain-binding protein (BiP), belongs to the heat shock protein 70 group and is located in the ER lumen. GRP78 was one of the best characterized ER chaperone proteins and its synthesis can be stimulated by a variety of environmental and physiological stress conditions that perturb ER function and homeostasis (6). GRP78 is currently regarded as the master regulator of the unfolding protein response (UPR) pathway (7), which may participate in mediate neuronal cell death after traumatic brain injury (8).

Caspase-12 is localized to the ER and has been shown to be activated by ER stress, including disruption of ER calcium homeostasis and accumulation of excess proteins in the ER $(9,10)$. Caspase-12 is also the key molecule in ER-associated apoptosis and activated caspase-12 can activate downstream apoptosis executioners, such as caspase-3, leading to apoptosis (11).

Amygdala, one of the key regions in the limbic system of the brain, has been recognized as a crucial brain structure involved in fear, rage and emotional memory $(12,13)$. Amygdala is usually divided into three distinct nuclear subgroups: Central nucleus, corticomedial nucleus and basolateral nucleus (14). Among these different nuclear sub-groups, the basolateral nucleus is the largest nucleus of the amygdaloid complex (15), which is a putative site of emotional memory and regulation of anxiety $(16,17)$. Thus, the present study focused on changes of the basolateral nucleus.

Single prolonged stress (SPS) (18) was shown to induce enhanced inhibition of the hypothalamo-pituitary-adrenal (HPA) axis, which is a putative neuroendocrinological hallmark of PTSD (19-21). Subsequently, SPS paradigms 
were extensively developed and employed in the investigation of PTSD $(22,23)$. The present study investigated the effects of SPS on the function of the ER by detecting GRP78 and caspase 12 in the amygdala of rats, and aimed to examine whether there was a link between an established rat model of PTSD and the ER of amygdala neurons. The findings revealed part of the pathogenesis and provided novel insight into the mechanism of how amygdala may participate in PTSD.

\section{Materials and methods}

Experimental animals. Eighty male Wistar rats, aged 7 or 8 weeks at the start of the study, weighing approximately 150-160 g, were supplied by the Animal Experimental Center, China Medical University. Rats were individually housed in an air-conditioned room $\left(22 \pm 1^{\circ} \mathrm{C}\right.$ and $55 \pm 5 \%$ humidity $)$ on a 12 -h light/dark schedule with free access to food and water.

The rats were raised in the laboratory for at least 7 days prior to conducting the experiment. Experiments were performed in accordance with the National Institute of Health Guide for the care and use of laboratory animals, and approved by the Welfare \& Ethics Committee of Experimental Animals (China Medical University, Shenyang, China). All efforts were made to reduce the number of animals used and to minimize animal suffering during the experiment.

Model establishment and grouping. Animals were divided randomly into four groups: 1) Control group; 2) SPS $1 \mathrm{~d}$ (1 day) group; 3) SPS 7 d (7 day) group; and 4) SPS 14 d (14-day) group. Control animals remained in their home cages with no handling for 7 or 14 days and were sacrificed at the same time as the SPS groups. SPS 1-, 7-and 14-day groups refer to the time after exposure to SPS. SPS rats underwent the SPS procedure on the first day. The SPS protocol was based on a combined plural stress paradigm $(24,25)$ : Immobilization (compression with plastic bags) for $2 \mathrm{~h}$, forced swimming in a clear acrylic cylinder for $20 \mathrm{~min}\left(24 \pm 1^{\circ} \mathrm{C}\right)$, rest for $15 \mathrm{~min}$, followed by ether anesthesia (until consciousness was lost).

Perfusion-based sections. Rats of the normal control and SPS groups were prepared via left ventricle perfusion and fixation (26) with $200 \mathrm{ml}$ of pre-cooled heparinized $0.9 \%$ saline, followed by $300 \mathrm{ml}$ of $4 \%$ paraformaldehyde in $0.01 \mathrm{M}$ phosphate-buffered saline (PBS) $(\mathrm{pH} 7.2-7.4)$. Brains were rapidly removed and post-fixed in the same fixative for 4-6 h at $4^{\circ} \mathrm{C}$, and were immersed in a $20 \%$ sucrose solution in $0.01 \mathrm{M}$ phosphate buffer (PB; pH 7.4) at $4^{\circ} \mathrm{C}$ thereafter. Samples were snap-frozen in liquid nitrogen and $12-\mu \mathrm{m}$ coronal sections were prepared for morphological studies.

Immunofluorescence analysis of GRP78. The sections of the normal control and SPS groups were treated with $5 \%$ bovine serum albumin (BSA; Roche Co., Shanghai, China) and $0.3 \%$ Triton X-100 (Sigma-Aldrich, St. Louis, MO, USA) in PBS for $30 \mathrm{~min}$ to block non-specific staining at room temperature (RT). Endogenous peroxidase was inactivated with $3 \% \mathrm{H}_{2} \mathrm{O}_{2}$ in double distilled $\mathrm{H}_{2} \mathrm{O}$ for $5 \mathrm{~min}$ at room temperature. The sections were then incubated with GRP78 mouse monoclonal antibody (A-10) (1:200 dilution; cat. no. sc-376768; Santa Cruz Biotechnology, Inc., Dallas, TX, USA) in 2\% BSA-PBS overnight at $4^{\circ} \mathrm{C}$. After being washed with PBS three times, the sections were incubated with fluorescein isothiocyanate goat anti-mouse monoclonal immunoglobulin G (IgG) (1:50 dilution; cat. no. BA1101; Boster Biological Technology, Wuhan, China) for $2 \mathrm{~h}$ at room temperature. To assess non-specific staining, several sections in every experiment were incubated in buffer without primary antibody. Slices were then mounted with glycerin and observed by fluorescence microscopy (BX61+DP71; Olympus Corp., Tokyo, Japan).

Fifteen slides were randomly selected from each group. In each slide, five visual fields in the basolateral amygdala were randomly selected (magnification, x400). The optical density (OD) of GRP78-immunopositive cells in each field was recorded to evaluate the average OD.

Western blot analysis of GRP 78 and caspase 12. Rats of the normal control and SPS groups were rapidly decapitated following inhalation anesthesia with ether, and the brains were removed and immediately placed in a dish standing on crushed ice. The basolateral amygdala was then dissected out according to the atlas of rats (27) using a stereomicroscope (SZX12; Olympus Corp.) and washed twice with 0.01 M PBS $(\mathrm{pH} 7.2-7.4)$ at $4^{\circ} \mathrm{C}$. Then the samples were homogenized with a sample buffer containing $200 \mathrm{mM}$ tris-buffered saline (TBS), pH 7.5, 4\% SDS, 20\% glycerol, 10\% 2-mercaptoethanol and were denatured by boiling for $3 \mathrm{~min}$. The protein fraction $(50 \mu \mathrm{g} /$ lane) prepared from each sample was separated by $12 \%(\mathrm{w} / \mathrm{v})$ gradient SDS-PAGE and electroblotted to a polyvinylidene difluoride (PVDF) membrane (Millipore, Bedford, MA, USA) from the gel using a semi-dry blotting apparatus (Bio-Rad Laboratories, Inc., Hercules, CA, USA).

The membrane was blocked with $5 \%$ skimmed milk powder and $0.05 \%$ Tween-20 (KeyGen Biotech. Co., Ltd., Nanjing, China) in TBS at room temperature for $2 \mathrm{~h}$ and incubated with GRP78 mouse monoclonal antibody (A-10) (1:200 dilution; cat. no. sc-376768; Santa Cruz Biotechnology, Inc.,) or caspase 12 rabbit polyclonal antibody (1611) (1:500 dilution; cat. no. sc-21747; Santa Cruz Biotechnology, Inc.) overnight at $4^{\circ} \mathrm{C}$.

Blots were washed three times with TBST and then incubated with horseradish peroxidase-conjugated goat anti-mouse (1:400; cat. no. sc-2073) or goat anti-rabbit (1:400; cat. no. sc-2040) IgG secondary antibodies for $2 \mathrm{~h}$ at room temperature and washed with TBST. After the incubation, the PVDF membrane was washed three times with TBST prior to visualization by enhanced chemiluminescence (ECL; KeyGen Biotech. Co., Ltd.). To confirm equal protein loading, the same blots were re-incubated with antibodies specific for $\beta$-actin (1:100 dilution; mouse monoclonal; cat. no. BM0627; Boster Biological Technology), which were detected using ECL. The OD was analyzed using the Gel Image Analysis System (Tanon 2500R; Tanon, Shanghai China). The relative expression levels of GRP78 and caspase 12 were determined by calculating the OD ratio of GRP78/ $\beta$-actin and caspase $12 / \beta$-actin.

Semiquantitative reverse transcription-polymerase chain reaction (RT-PCR) for detection of GRP78 and caspase 12 . Total mRNA of each group was extracted from the basolateral amygdala according to the instructions of the TRIzol kit (Invitrogen Life Technologies, Carlsbad, CA, USA) and 
Table I. Primer sequences of GRP78, caspase 12 and $\beta$-actin.

\begin{tabular}{llll}
\hline Name & Upstream primer & Downstream primer & Product size (bp) \\
\hline GRP78 & 5'-TAATCAGCCCACCGTAACAATC-3' & 5'-ACCTCCCAGCTTCTCTTTATCT-3' & 385 \\
Caspase 12 & 5'-TGCCAATTCCGACAAACAGC-3' & 5'-TGCCGTCCCACATAAAGACC-3' & 512 \\
$\beta$-actin & 5'-ATCACCCACACTGTGCCCATC-3' & 5'-ACAGAGTACTTGCGCTCAGGA-3' & 542 \\
\hline
\end{tabular}
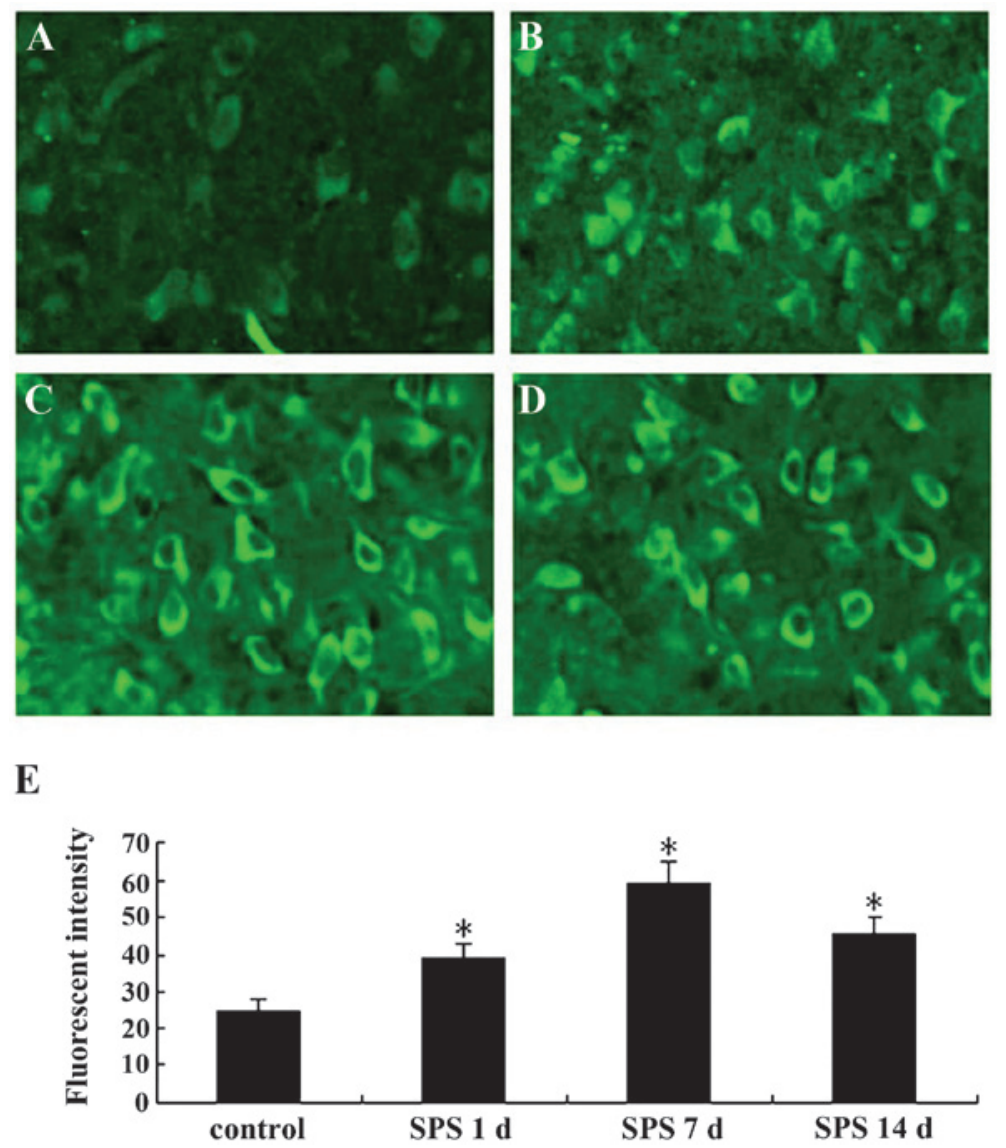

Figure 1. Immunohistochemical detection of glucose-regulated protein 78 expression in the basolateral amygdala of each group. (A) control group; (B) SPS $1 \mathrm{~d}$; (C) SPS 7 d; (D) SPS 14 d (A-D, magnification, x400). (E) Semi-quantitative analysis results of fluorescence intensity. Values are presented as the mean \pm standard error. "P<0.05 vs. control group. SPS, single prolonged stress.

$1 \mu \mathrm{g}$ of total RNA was reverse transcribed into cDNA. cDNA was amplified using an RNA PCR kit (AM Ver. 3.0; Takara Bio, Inc., Otsu, Japan). The primers were designed and synthesized by Shenggong Biotech Company (Shanghai, China) according to the serial number from GenBank and are shown in Table I. The reaction was started at $94^{\circ} \mathrm{C}$ for 2 min, amplification comprised 30 cycles of $30 \mathrm{sec}$ at $94^{\circ} \mathrm{C}$, $30 \mathrm{sec}$ at $56^{\circ} \mathrm{C}, 40 \mathrm{sec}$ at $72^{\circ} \mathrm{C}$ (for GRP78) or 27 cycles of $30 \mathrm{sec}$ at $94^{\circ} \mathrm{C}, 30 \mathrm{sec}$ at $50^{\circ} \mathrm{C}, 45 \mathrm{sec}$ at $72^{\circ} \mathrm{C}$ (for caspase-12) and ended with 5 min extension at $72^{\circ} \mathrm{C}$, using XP Thermal Cycler (Hangzhou Bioer Technology Co., Ltd., Hangzhou, China). $\beta$-actin mRNA used as an internal control and was co-amplified with GRP78- or caspase 12- mRNA. The products were separated by electrophoresis on a $1.2 \%$ agarose gel, and the density of each band was analyzed using the Gel Image Analysis System (Tanon 2500R; Tanon). The levels of GRP78- and caspase 12-mRNA were determined by calculating the density ratio of GRP78 $\mathrm{mRNA} / \beta$-actin mRNA or caspase 12 mRNA/ $\beta$-actin mRNA.

Assessment of morphological changes of the ER using transmission electron microscopy (TEM). Rats of each group were perfused with pre-cold heparinized $0.9 \%$ saline, followed by 0.01 M PBS ( $\mathrm{pH} 7.2-7.4$ ) containing $4 \%$ paraformaldehyde and $2.5 \%$ glutaraldehyde. The brain was removed and dissected on ice, followed by $4-6 \mathrm{~h}$ of post-fixation in the same fixative at $4^{\circ} \mathrm{C}$. The basolateral amygdala was dissected by using a stereomicroscope and cut into blocks of $\sim 1 \mathrm{~mm}^{3}$. The blocks were post-fixed in $1 \%$ osmium tetroxide for $2 \mathrm{~h}$ at $4^{\circ} \mathrm{C}$. They were rinsed in 0.01 M PBS ( $\mathrm{pH} 7.4$ ) several times, dehydrated in a graded series (20-100\%) of ethanol and then in acetone, infused with Epon 812, and finally polymerized in pure Epon 812 (Serva, New York, NY, USA) at $65^{\circ} \mathrm{C}$ for $72 \mathrm{~h}$. The basolateral amygdala was localized on semi-thin sections. Ultra-thin sections were cut on 


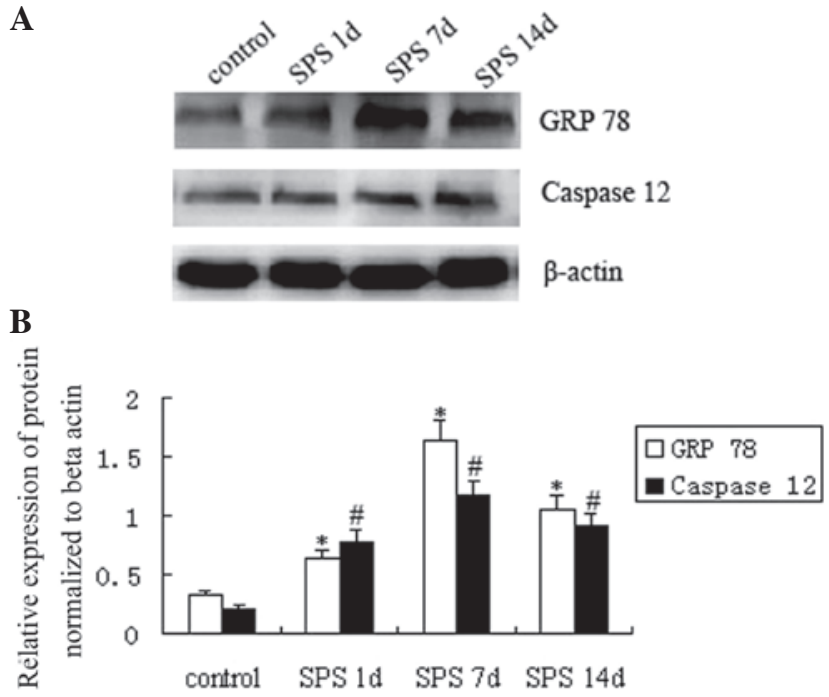

Figure 2. (A) Presentation of bands showing GRP78, caspase 12 and $\beta$-actin protein levels in the basolateral amygdala at the indicated time-points after SPS stimuli by western blot analysis. (B) Relative quantitative expression levels of GRP 78 and caspase 12. Values are presented as the mean \pm standard error. ${ }^{*} \mathrm{P}<0.05$ vs. control group of GRP $78,{ }^{\prime \prime} \mathrm{P}<0.05$ vs. control group of caspase 12. GRP, glucose-regulated protein; OD, optical density.

A
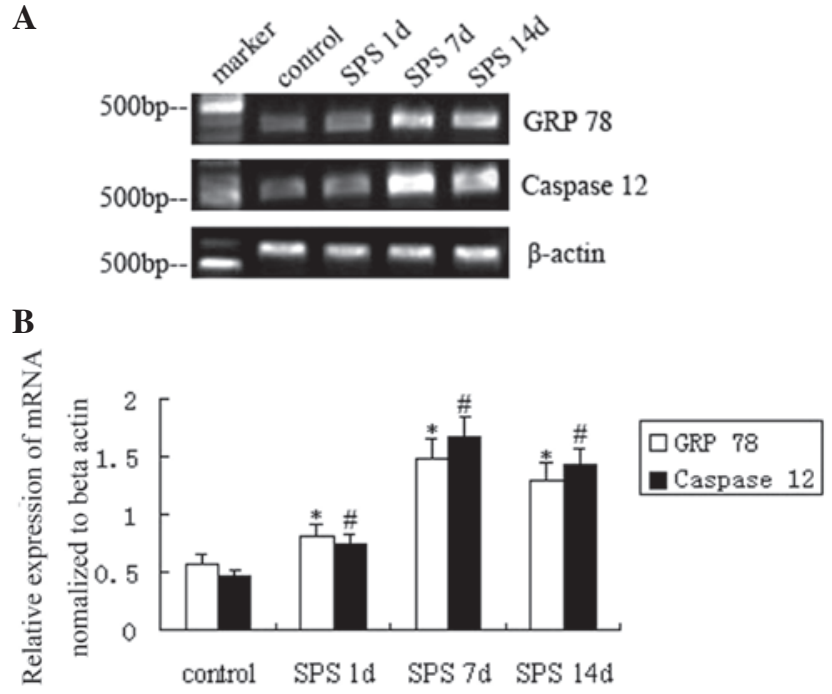

Figure 3. (A) Representative gels showing cDNA bands of GRP 78, caspase 12 and $\beta$-actin in the basolateral amygdala and (B) relative expression of GRP78 and caspase 12 mRNA at the indicated time-points after SPS stimuli. Values are presented as the mean \pm standard error. ${ }^{*} \mathrm{P}<0.05$ vs. control group of GRP $78,{ }^{\#} \mathrm{P}<0.05$ vs. control group of caspase 12 . GRP, glucose-regulated protein; OD, optical density.

an ultramicrotome, collected on copper grids, and stained with $4 \%$ uranyl acetate and lead citrate. A minimum of 5 sections comprising 250 cells from each basolateral amygdala were studied with transmission electron microscopy (TEM-1200EX; 80KV; Jeol Ltd., Tokyo, Japan).

Statistical analysis. All values were expressed as the mean \pm standard error. Data among groups were analyzed by one-way analysis of variance using SPSS 13.0 software (SPSS, Inc. Chicago, IL, USA). $\mathrm{P}<0.05$ was considered to indicate a statistically significant difference between values.
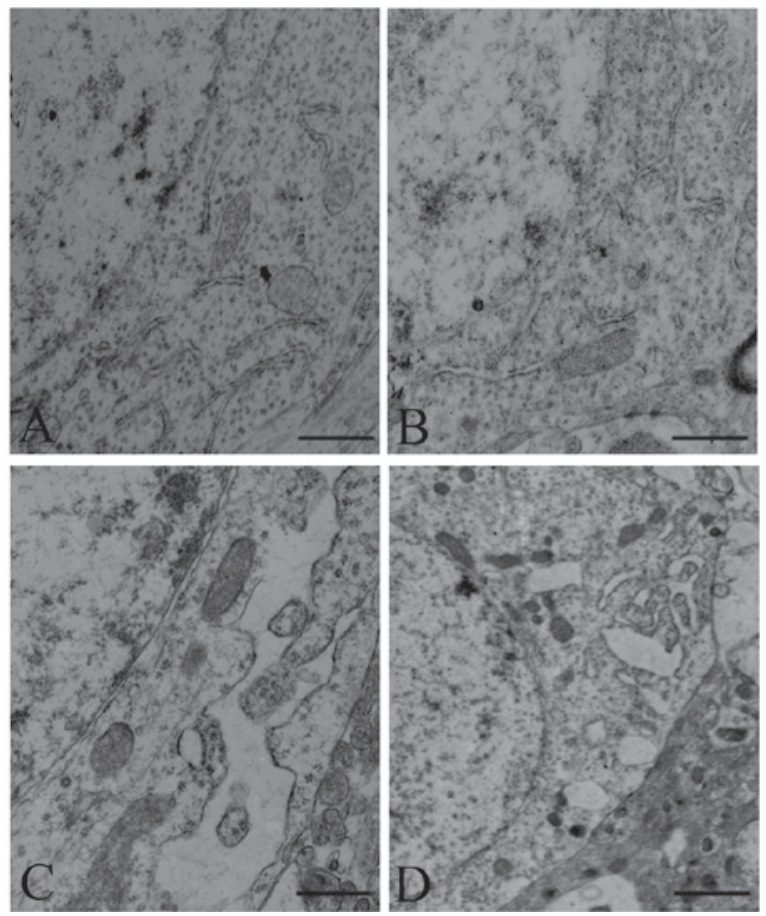

Figure 4. Transmission electron microscopic analysis of the endoplasmic reticulum of basolateral amygdala neurons in rats of each group. (A) control group; (B) SPS 1 d; (C) SPS 7 d; (D) SPS 14 d) (scale bars, $1 \mu \mathrm{m}$ ). SPS, single prolonged stress.

\section{Results}

SPS increases GRP78 and caspase 12 levels in the basolateral amygdala. The immunofluorescence staining results are shown in Fig. 1. The GRP78 protein was located in the cytoplasm (Fig. 1A-D). In the normal control group, the fluorescent intensity of GRP78-positive cells was low, while that in SPS rats was significantly higher and was highest at 7 days after exposure to SPS (Fig. 1E) $(\mathrm{P}<0.01)$.

In the western blots, immunoreactive signals for GRP78, caspase 12 and $\beta$-actin appeared at $78 \mathrm{kDa}, 50 \mathrm{kDa}$ and $42 \mathrm{kDa}$, respectively (data not shown), and the mean value of band densities of the control group was set as $100 \%$. Data were expressed as normalized optical density. GRP78 and caspase 12 protein levels in the basolateral amygdala region of the different groups are presented in Fig. 2A. The results showed that SPS exposure resulted in a significant change of GRP78 and caspase 12. GRP78 expression was upregulated 1 day after SPS stimulation as compared with that in the control group, was highest in the SPS $7 \mathrm{~d}$ group and was decreased thereafter in the SPS $14 \mathrm{~d}$ group, while still being higher than that in the SPS $1 \mathrm{~d}$ group $(\mathrm{P}<0.05)$. Similarly, caspase 12 protein expression in the SPS model groups also showed a significant upregulation in comparison with control rats, was highest in the SPS $7 \mathrm{~d}$ and then declined in the SPS $14 \mathrm{~d}$ group (Fig. 2B) $(\mathrm{P}<0.05)$.

For semiquantitative PCR, levels of GRP 78 and caspase-12 mRNA were normalized to $\beta$-actin mRNA levels. In analogy with the protein levels, mRNA levels of GRP 78 and caspase-12 gradually increased after SPS stimulation compared with those in the control group and were highest at SPS $7 \mathrm{~d}(\mathrm{P}<0.01)$ (Fig. 3). 
SPS causes morphological changes of the ER of amygdala neurons. As shown in Fig. 4A, the intracellular ER of amygdala neurons exhibited a normal structure in the control rats (Fig. 4A). Mild distension of the ER was observed in the SPS $1 \mathrm{~d}$ group (Fig. 4B). A tumescent ER, ER vacuolization and degranulation of ER were observed in the SPS $7 \mathrm{~d}$ group (Fig. 4C) $(\mathrm{P}<0.05)$. Furthermore, as shown in Fig. 4D, the ER of amygdala neurons also exhibited an abnormal structure in the SPS 14 d group.

\section{Discussion}

PTSD is thought to involve a disfunction in response to fear-associated stimuli. Four major types of characteristic symptoms of PTSD are re-experiencing, avoidance, numbing and hyperarousal (1). The specific role of amygdala in the processing of threat-associated stimuli, in particular anger and fear, has been well documented based on investigations on animals and humans (28-30). Numerous lines of evidence have implicated the basolateral amygdala (BLA) as a substrate for stress-associated modulation of memory (31). Therefore, the present study focused on observing SPS-induced changes in the basolateral nucleus.

A previous study has demonstrated that ER stress is closely associated with several diseases, including neuronal cell injury (32), Alzheimer's disease (33) and Parkinson's disease (34). In the present study, changes in the levels of ER stress protein GRP78 and ER-resident caspase-12 in the amygdala of rats were detected in order to identify whether ER stress is involved in PTSD. GRP78, the master regulator of the UPR pathway and a molecular chaperone in the ER that provides cytoprotection in response to cellular stresses, was significantly upregulated in the rats after exposure to SPS stimuli, which may have resulted in dysfunction of the ER. Caspase 12 was also significantly upregulated after SPS stimulation. The results of the morphological evaluation showed that tumescent ER, ER vacuolization and degranulation of the ER were present in the SPS groups. In conclusion, the results indicated that GRP78 and caspase 12 were significantly upregulated and morphological changes in the amygdala of rats were present after exposure to SPS. The possible reason for this is that SPS stimuli induced the activation of the UPR pathway, and the accumulation of unfolded or misfolded proteins led to ER dysfunction of amygdala neurons, which may play an important role in the pathobiological basis for the abnormality of affect and behavior induced by PTSD.

One limitation of the present study is that it did not examine whether apoptosis was induced via the ER pathway in the amygdala, although a previous study by our group has demonstrated that SPS induced more apoptotic cells and increased the apoptotic rate in the amygdala of rats after exposure to SPS compared with that in the normal control group (35). Further study will examine whether apoptosis activated by ER stress participates in mechanisms of PTSD. The results of the present study revealed increases in the levels of ER stress protein GRP78 and ER-resident caspase-12; however, their pathophysiological roles in PTSD remain elusive.

At present, the pathogenesis of PTSD is not yet entirely clear. PTSD may cause a series of biochemical abnormalities and dysfunction of the amygdala, which leads to dysfunction of the brain (36). The present study has shed light on the cellular mechanisms of ER stress in the amygdala and their participation in the pathogenesis of PTSD, which may lead to the development of novel treatments for PTSD. Further investigation into the molecular mechanisms of how the ER regulates neuronal function and the exact cellular pathway should also be elucidated. Thus, the pathogenesis of PTSD requires further investigation.

\section{Acknowledgements}

The present study was supported by a grant from the National Natural Science Foundation of China (no. 31200772) and the Doctoral Program Research Foundation of Higher Education of China (no. 20132104110021). The authors would like to thank the anonymous reviewers for their valuable comments on how to improve the quality of the paper.

\section{References}

1. American Psychiatric Association: Diagnostic and Statistical Manual of Mental Disorders, 4th ed. DSM-IV. American Psychiatric Press Washington DC, 1994.

2. Corbett EF and Michalak M: Calcium, a signaling molecule in the endoplasmic reticulum. Trends Biochem Sci 25: 307-311, 2000.

3. Nakamura K, Bossy-Wetzel E, Burns K, et al: Changes in endoplasmic reticulum luminal environment affect cell sensitivity to apoptosis. J Cell Biol 150: 731-740, 2000.

4. Su HL, Liao CL and Lin YL: Japanese encephalitis virus infection initiates endoplasmic reticulum stress and an unfolded protein response. J Virol 76: 4162-4171, 2002.

5. Rao RV and Bredesen DE: Misfolded proteins, endoplasmic reticulum stress and neurodegeneration. Curr Opin Cell Biol 16: 653-662, 2004

6. Lee AS: The ER chaperone and signaling regulator GRP78/Bip as a monitor of endoplasmic reticulum stress. Methods 35: 373-381, 2005.

7. Radley JJ, Arias CM and Sawchenko PE: Regional differentiation of the medial prefrontal cortex in regulating adaptive responses to acute emotional stress. J Neurosci 26: 12967-12976, 2006.

8. Martinez JA, Zhang Z, Svetlov SZ, et al: Calpain and caspase processing of caspase-12 contribute to the ER stress-induced cell death pathway in differentiated PC12 cells. Apoptosis 15: 1480-1493, 2010.

9. Momoi T: Caspases involved in ER stress-mediated cell death. J Chem Neuroanat 28: 101-105, 2004.

10. Nakagawa T, Zhu H, Morishima N, et al: Caspase-12 mediates endoplasmic-reticulum-specific apoptosis and cytotoxicity by amyloid- $\beta$. Nature 403: 98-103, 2000.

11. Liu LQ, Fan ZQ, Tang YF and Ke ZJ: The Resveratrol attenuates ethanol-induced hepatocyte apoptosis via inhibiting ER-related caspase-12 activation and PDE activity in vitro. Alcohol Clin Exp Res 38: 683-693, 2014.

12. McGaugh JL and Cahill L: Interaction of neuromodulatory systems in modulating memory storage. Behav Brain Res 83: 31-38, 1997.

13. LeDoux JE: Emotion: clues from the brain. Annua Rev Psychol 46: 209-235, 1995.

14. Harding AJ, Stimson E, Henderson JM, et al: Clinical correlates of selective pathology in the amygdala of patients with Parkinson's disease. Brain 125: 2431-2445, 2002.

15. Sims KS and Williams RS: The human amygdaloid complex: a cytologic and histochemical atlas using Nissl, myelin, acetylcholinesterase and nicotinamide adenine dinucleotide phosphate diaphorase staining. Neuroscience 36: 449-472, 1990.

16. Davis M: The role of the amygdala in emotional learning. Int Rev Neurobiol 36: 225-266, 1994.

17. McGaugh JL, Mclntyre CK and Power AE: Amygdala modulation of memory consolidation: interaction with other brain systems. Neurobiol Learn Mem 78: 539-552, 2002.

18. Liberzon I, Krstov M and Young EA: Stress-restress: effects on ACTH and fast feedback. Psychoneuroendocrinology 22: 443-453, 1997. 
19. Stein MB, Yehuda R, Koverola C, et al: Enhanced dexamethasone suppression of plasma cortisol in adult women traumatized by childhood sexual abuse. Biol Psychiatry 42: 680-686, 1997.

20. Yehuda R: Biology of posttraumatic stress disorder. J Clin Psychiatry 62: 41-46, 2001.

21. Yehuda R: Neuroendocrine aspects of PTSD. Handb Exp Pharmacol 169: 371-403, 2005.

22. Khan S and Liberzon I: Topiramate attenuated exaggerated acoustic startle in an animal model of PTSD.Psychopharmacology (Berl) 172: 225-229, 2004.

23. Iwamoto Y, Morinobu S, Takahashi T, et al: Single prolonged stress increases contextual freezing and the expression of glycine transporter 1 and vesicle-associated membrane protein 2 mRNA in the hippocampus of rats. Prog Neuropsychopharmacol Biol Psychiatry 31: 642-651, 2007.

24. Takahashi T, Morinobu S, Iwamoto Y, et al: Effect of paroxetine on enhanced contextual fear induced by single prolonged stress in rats. Psychopharmacology (Berl) 189: 165-173, 2006.

25. Kohda K, Harada K, Kato K, et al: Glucocorticoid receptor activation is involved in producing abnormal phenotypes of single-prolonged stress rats: a putative post-traumatic stress disorder model. Neuroscience 148: 22-33, 2007.

26. Liu HY: Technical operations and its common problems of perfusion fixation in mice. Qiqihaer Yixueyuan Xuebao 27: 1341, 2006.

27. Paxinos G and Watson C: The Rat Brain in Stereotaxic Coordinates. 4th edition Academic Press, 1998.
28. Derntl B, Windischberger C, Robinson S, et al: Amygdala activity to fear and anger in healthy young males is associated with testosterone. Psychoneuroendocrinology 34: 687-693, 2009.

29. McGauqh JL: The amygdala modulates the consolidation of memories of emotionally arousing experience. Annu Rev Neurosci 27: 1-28, 2004.

30. Cahill L and McGaugh JL: Mechanisms of emotional arousal and lasting declarative memory. Trends Neurosci 21: 294-299, 1998.

31. Chavez CM, McGaugh JL and Weinberger NM: The basolateral amygdala modulates specific sensory memory representations in the cerebral cortex. Neurobiol Learnand Mem 91: 382-392, 2009.

32. Paschen W and Frandsen A: Endoplasmic reticulum dysfunction-a common denominator for cell injury in acute and degenerative diseases of the brain. J Neurochem 79: 719-725, 2001.

33. Katayama T, Imaizumi K, Sato N, et al: Presenilin-lmutations downregulate the signaling pathway of the unfolded-protein response. Nat Cell Biol 1: 479-485, 1999.

34. Imai $\mathrm{Y}$, Soda $\mathrm{M}$, Inoue $\mathrm{H}$, et al: An unfolded putative transmembrane polypeptide, which can lead to endoplasmic reticulum stress, is a substrate of Parkin. Cell 105: 891-902, 2001.

35. Ding JL, Han F and Shi YX: Single-prolonged stress induces apoptosis in the amygdala in a rat model of post-traumatic stress disorder. J Psychiatr Res 44: 48-55, 2010.

36. Xiao B, Han F and Shi YX: Dysfunction of $\mathrm{Ca}^{2+} / \mathrm{CaM}$ kinase IIalpha cascades in the amygdala in post-traumatic stress disorder. Int J Mol Med 24: 795-799, 2009. 